\title{
Quantum dynamics of a resonator driven by a superconducting single-electron transistor: a solid-state analogue of the micromaser
}

\author{
D.A. Rodrigues, J. Imbers and A.D. Armour \\ School of Physics and Astronomy, University of Nottingham, Nottingham NG7 2RD, United Kingdom
}

\begin{abstract}
We investigate the behavior of a quantum resonator coupled to a superconducting single-electron transistor tuned to the Josephson quasiparticle resonance and show that the dynamics is similar in many ways to that found in a micromaser. Coupling to the SSET can drive the resonator into non-classical states of self-sustained oscillation via either continuous or discontinuous transitions. Increasing the coupling further leads to a sequence of transitions and regions of multistability.
\end{abstract}

PACS numbers: $85.85 .+\mathrm{j}, 85.35 . \mathrm{Gv}, 74.78 . \mathrm{Na}$

Systems where a mesoscopic conductor such as a single-electron transistor is coupled to a nanomechanical resonator have been studied intensively because the current through the conductor can be extremely sensitive to the motion of the resonator and hence may be used to monitor its position with almost quantum-limited precision 1, 2, 3, 4]. Furthermore, where either the coupling between the electrons and the resonator is non-linear 5] or the electronic transport occurs via a resonance [4], dynamic instabilities in the resonator can occur leading to self-sustained oscillations. The way a nanomechanical resonator can be driven into states of finite amplitude oscillation by successive interactions with a current of electrons in a conductor parallels the behavior of quantum optical systems, such as the micromaser, in which an electromagnetic cavity is pumped by interactions with a steady stream of individual two-level atoms [ $[$ ] . This contrasts with a standard laser (a nanomechanical version of which was envisioned in 7]) where an oscillator interacts simultaneously with many two-level systems.

In a superconducting single-electron transistor (SSET) transport can occur via resonant processes involving both coherent motion of Cooper pairs and incoherent quasiparticle tunneling, the simplest of which is the Josephson quasiparticle (JQP) resonance [8]. In the vicinity of a JQP resonance, the dynamics of a resonator coupled linearly to the SSET is very sensitive to the bias point [3, 4, 9]. For bias points on one side of the resonance, the SSET acts on the resonator like a thermal bath and its current can monitor the position of the resonator with exquisite sensitivity. In contrast, biasing on the opposite side of the JQP resonance can drive the resonator into states of self-sustained oscillation [4].

In this Letter we explore the quantum dynamics of a resonator coupled to a SSET and show that it is analogous to that of a micromaser. Less noisy than a laser, a micromaser [6, 10] can generate number-squeezed states of the cavity and exhibits not a single threshold transition, but a series of transitions between different dynamical states. Although the SSET-resonator system and micromaser differ in the details of the interactions between their respective sub-components, we find a num- (a)
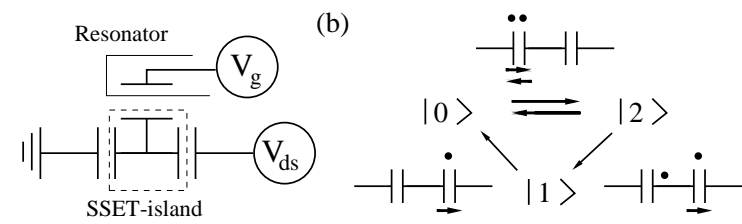

FIG. 1: (a) Schematic diagram of the SSET-resonator system. The SSET consists of a superconducting island linked by tunnel junctions to superconducting leads, across which a voltage $V_{d s}$ is applied. (b) Charge processes involved in the JQP current cycle (details are given in the text).

ber of important similarities in their dynamics, many of which first arise when the resonator is sufficiently fast to match the time-scale of the electrical transport. Previous theoretical studies of this system have concentrated on the limit of a slow resonator [4, 9] as it is only this limit which has so far been explored in experiments on nanomechanical resonators coupled to a SSET [2, 3]. The much faster resonator speeds which we also consider here might be achieved by making a smaller 11] mechanical resonator. However, it should also be possible to use a superconducting resonator (e.g. a stripline resonator [12]) for which much higher frequencies are practical.

The SSET-resonator system we consider is shown schematically in Fig. 17. A mechanical resonator acts as a voltage-gate with a position dependent capacitance and the coupling is controlled by varying the voltage applied to it (an analogous, though fixed, electrostatic coupling arises for a superconducting stripline resonator [12]). The SSET is assumed to be biased close to a JQP resonance [8] where only three charge states of the SSET island are relevant. Current flows in a cycle (Fig. 10): coherent Josephson tunneling between the left lead and the island produces a superposition of island charge-states $|0\rangle$ and $|2\rangle$, this is then followed by two quasiparticle tunneling events between the island and the right lead which take the SSET island from state $|2\rangle$ to state $|1\rangle$ and finally back to state $|0\rangle$. The resonator is modeled as a single-mode quantum harmonic oscillator with mass $m$ and angular frequency $\omega$. For a weak electrostatic interaction between the resonator and the SSET, the cou- 
pling between the resonator position and the number of excess charges on the SSET island is linear [4, 9]. The SSET-resonator system can therefore be described by the Hamiltonian

$$
\begin{aligned}
H= & \Delta E|2\rangle\langle 2|-\frac{E_{J}}{2}(|0\rangle\langle 2|+| 2\rangle\langle 0|)+\hbar \omega a^{\dagger} a \\
& +x_{s}\left(\hbar \omega^{3} m / 2\right)^{1 / 2}\left(a^{\dagger}+a\right)(|1\rangle\langle 1|+2| 2\rangle\langle 2|),
\end{aligned}
$$

where $a$ is the annihilation operator for the resonator, $\Delta E$ is the electrostatic energy difference between the island states $|0\rangle$ and $|2\rangle, x_{s}$ is the displacement in the equilibrium position of the resonator when one electronic charge is added to the SSET island [9] and $E_{J}$ the Josephson energy of the superconductors. The system can be considered to be in the weak-coupling limit when the voltage applied across the SSET is much larger than the coupling energy, $\kappa=m \omega^{2} x_{s}^{2} / e V_{d s} \ll 1[9]$.

The system evolves coherently under the action of the Hamiltonian, but dissipation arises from two sources: quasiparticle tunneling in the SSET and the resonator's surroundings. Thus the master equation for the SSETresonator density matrix, $\rho(t)$, derived using the BornMarkov approach [8], takes the form

$$
\dot{\rho}=\mathcal{L} \rho=-\frac{i}{\hbar}[H, \rho]+\mathcal{L}_{\text {leads }} \rho+\mathcal{L}_{\text {damping }} \rho,
$$

where the dissipative terms arising from the resonator's surroundings and from quasiparticle tunneling are described by the terms $\mathcal{L}_{\text {damping }}$ and $\mathcal{L}_{\text {leads }}$ respectively. The resonator's surroundings are assumed to act like a thermal bath which we describe using a Liouvillian [13],

$$
\begin{aligned}
\mathcal{L}_{\text {damping }} \rho= & -\frac{\gamma_{\text {ext }}}{2}(\bar{n}+1)\left(a^{\dagger} a \rho+\rho a^{\dagger} a-2 a \rho a^{\dagger}\right) \\
& -\frac{\gamma_{\text {ext }}}{2} \bar{n}\left(a a^{\dagger} \rho+\rho a a^{\dagger}-2 a^{\dagger} \rho a\right),
\end{aligned}
$$

which is guaranteed to preserve the positivity of the density matrix. The temperature of the external bath is parameterised by the average number of quanta, $\bar{n}$, that the resonator would have were it in thermal equilibrium with the bath and the resonator-bath coupling is given by a damping rate $\gamma_{e x t}$. The tunneling of quasiparticles from the island to the left lead is described by [8]

$$
\begin{aligned}
\mathcal{L}_{\text {leads }} \rho= & -\frac{\Gamma}{2}\left[\{|2\rangle\langle 2|+| 1\rangle\langle 1|, \rho\}_{+}\right. \\
& -2(|1\rangle\langle 2|+| 0\rangle\langle 1|) \rho(|2\rangle\langle 1|+| 1\rangle\langle 0|)],
\end{aligned}
$$

where $\Gamma$ is the quasiparticle tunneling rate. This is a simplified expression in which we have neglected differences between the quasiparticle rates for the two processes, their variation with bias point and temperature dependence [4, 8, 9]. We have also neglected the dependence of the quasiparticle tunneling rates on the resonator position as this is much less important than the coherent electro-mechanical coupling in the Hamiltonian

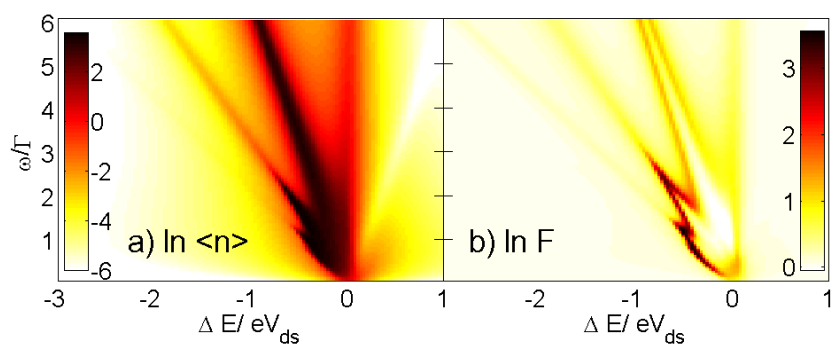

FIG. 2: (Color online) Steady state properties of the resonator as a function of $\Delta E$, and resonator speed, $\omega / \Gamma$ : (a) average occupation number of the resonator, $\langle n\rangle$; (b) Fano factor of the resonator, both plotted on a natural-log scale, with $\kappa=$ $0.01, \gamma_{e x t} / \Gamma=0.002$, and $\bar{n}=0$.

(Eq. 1) [4, 9, 14]. These simplifications allow us to capture the essential phenomenology of the system using a relatively compact model. Furthermore, simplified in this way our model of the SSET-resonator system close to the JQP resonance is dual to another system, that of a double quantum dot gated by a resonator [15].

The value of $\Delta E$ determines the detuning of the Cooper pairs from resonance and can be changed continuously by changing the applied gate voltage (this can be done independently of the coupling provided an additional, fixed, gate is used [3] ). The sign of $\Delta E$ determines the direction of (average) energy flow between the resonator and the SSET. For $\Delta E<0$ the state $|0\rangle$ has more energy than $|2\rangle$, hence when a Cooper pair tunnels onto the island it can pass some of its energy to the resonator (before quasiparticle decays occur), but when $\Delta E>0$ the situation is reversed and hence the Cooper pair can absorb energy from the resonator. This means that when $\Delta E>0$ the SSET damps the motion of the resonator, though because of the stochastic nature of the current, the resonator settles into a thermal-like steady state, not its ground state [4, 9]. However, when $\Delta E<0$ the transfer of successive Cooper pairs passing from lead to island can pump the resonator and drive its oscillation.

In order to explore the behavior of the system over a range of resonator speeds, from slow $(\omega / \Gamma \ll 1)$ to fast $(\omega / \Gamma \gg 1)$ we solve the master equation using a numerical method. There are a number of methods which can be employed to calculate the steady state of a master equation. We have made use of the numerical routines implemented in the Quantum Optics Toolbox [16] to calculate the steady state of the density matrix by evaluating the eigenfunction corresponding to the zero eigenvalue of the Liouvillian in Eq. 2(written in matrix form). Our method necessitates a truncation of the resonator's Hilbert space, which because of the need to ensure convergence, effectively translates into a lower limit on the value of the external damping we consider (we used a Fock state representation with up to 70 states).

The effect on the steady state of the resonator of vary- 


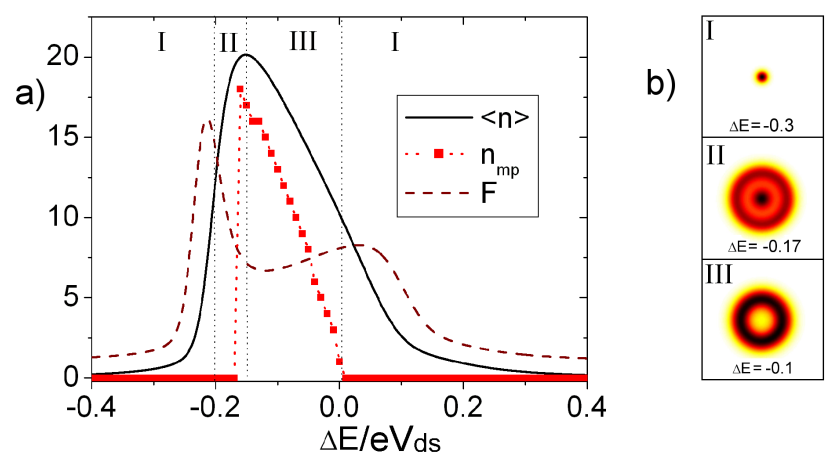

FIG. 3: (Color online)(a) Average occupation number and Fano factor of the resonator together with the most probable number state in the density matrix, $n_{m p}$, for $\omega / \Gamma=0.1$, $\gamma_{\text {ext }} / \Gamma=0.0005, \kappa=0.02$ and $\bar{n}=0$. (b) Examples of the resonator Wigner functions: I fixed point state, II bistable and III limit cycle. The regions in (a) corresponding to the three basic Wigner function topologies are also marked as I (fixed point), II (bistable) and III (limit cycle).

ing the detuning from resonance, $\Delta E$, for a range of resonators speeds, $\omega / \Gamma$, is shown in Fig. 2 where the average number of resonator quanta, $\langle n\rangle$ (with $n=a^{\dagger} a$ ), and the Fano factor, $F=\left(\left\langle n^{2}\right\rangle-\langle n\rangle^{2}\right) /\langle n\rangle$ are plotted. We assume (throughout) SSET parameters [9] $\Gamma=V_{d s} / e R_{J}$, $R_{J}=h / e^{2}$ and $E_{J}=h V_{d s} /\left(16 e R_{J}\right)$, where $R_{J}$ is the SSET junction resistance.

A more complete understanding of the resonator state is obtained from the Wigner transform of the reduced steady state density matrix of the resonator [5]. Although the Wigner function of the resonator is different in detail for every set of parameters in Fig. 2, it turns out that because of the weak SSET-resonator coupling there are just three different topologies which arise: a state in which the resonator fluctuates about a fixed point, a limit cycle state in which the resonator undergoes finite amplitude oscillations and a bistable state in which both the fixed point and limit cycle type states coexist (illustrated in Fig. (3).

The behavior of the resonator is simplest in the limits of a very fast $(\omega / \Gamma \gg 1)$ or very slow $(\omega / \Gamma \ll 1)$ resonator, where a wide separation of the SSET and resonator time-scales limits their mutual interaction. In contrast, when $\omega / \Gamma \sim 1$ the SSET and resonator interact most strongly and hence it is not surprising that it is in this regime that the most interesting features of the coupled dynamics first begin to emerge [17].

For $\omega / \Gamma \geq 1$, Fig. 2 shows that the transfer of energy between SSET and resonator is increasingly concentrated around a series of lines. These lines are points where $\Delta E= \pm j \hbar \omega$ with $j$ an integer (the current through the SSET also shows peaks at these values). When $\Delta E<0$, the resonator absorbs quanta of energy from the SSET leading to an enhancement of $\langle n\rangle$ and $F$. Because we are working in the weak coupling regime, the strongest effect occurs for $\Delta E=-\hbar \omega$, with progressively weaker signatures at $\Delta E=-2 \hbar \omega$ and $-3 \hbar \omega$, as the exchange of multiple quanta result from higher order processes. A dip in $\langle n\rangle$ for $\Delta E=+\hbar \omega$ is also visible though it becomes less pronounced as $\omega / \Gamma$ is increased. This dip occurs, despite the fact that we have assumed an external bath at zero temperature, as the noise arising from coupling to the SSET excites the resonator out of its ground state even for $\Delta E>0$. These results are consistent with calculations for a resonator coupled to a double quantum dot in this regime [15].

In the limit of a slow resonator, $\omega / \Gamma \ll 1$, variation of $\Delta E$ leads to two distinct transitions in the state of the resonator as is shown in Fig. 3. The transitions become sharper as the ratio of the SSET current to the relaxation rate $\gamma_{e x t}$ increases, which suggests that they can be thought of as non-equilibrium phase transitions in a 'thermodynamic limit' where this ratio diverges [10, 18]. The most probable number state in the density matrix, $n_{m p}$, provides a convenient order parameter of the system. For $\Delta E / e V_{d s} \gtrsim 0.4$, the resonator is very close to being in a thermal state and hence there is a single peak in the Wigner function, but as $\Delta E$ is decreased and approaches zero the resonator state broadens (deviating strongly from a thermal state), until for $\Delta E \simeq 0$ a limit cycle begins to appear. The transition to a limit cycle is continuous as the radius of the cycle (and $n_{m p}$ ) grows steadily from zero as $\Delta E$ is decreased. However, eventually (as $\Delta E$ becomes sufficiently negative) the system passes through a region of bistability where both the limit cycle and a central peak co-exist in the Wigner function before the limit cycle disappears entirely and only the central peak remains. This behavior implies a discontinuous transition and is marked by a jump in $n_{m p}$.

For a very slow resonator, the current flowing through the SSET decays monotonically to zero far from the center of the JQP resonance. This explains why the limit cycle eventually disappears as $\Delta E$ is decreased: the energy per unit time transferred to the resonator is proportional to the current and as this decays, the system will eventually be stabilized by the external damping.

A striking feature of the intermediate regime $(\omega / \Gamma \sim$ 1) in Fig. 2 is the drop in the Fano factor of the resonator which occurs in the limit cycle state. Remarkably, there is a region where the Fano factor falls below unity, implying that the resonator is driven into a number squeezed (i.e. non-classical) steady state. We attribute this squeezing to non-linear damping induced by the interaction with the SSET [4]. Squeezing of this kind is a characteristic of the micromaser [6, 10], but never occurs in the (more noisy) conventional laser [18]. As with the micromaser, we find that increasing the noise in the system (by increasing the temperature of the bath) rapidly washes out the squeezing effect.

The regime where $\omega / \Gamma \sim 1$ is also where further transitions beyond a simple limit cycle state first occur as 


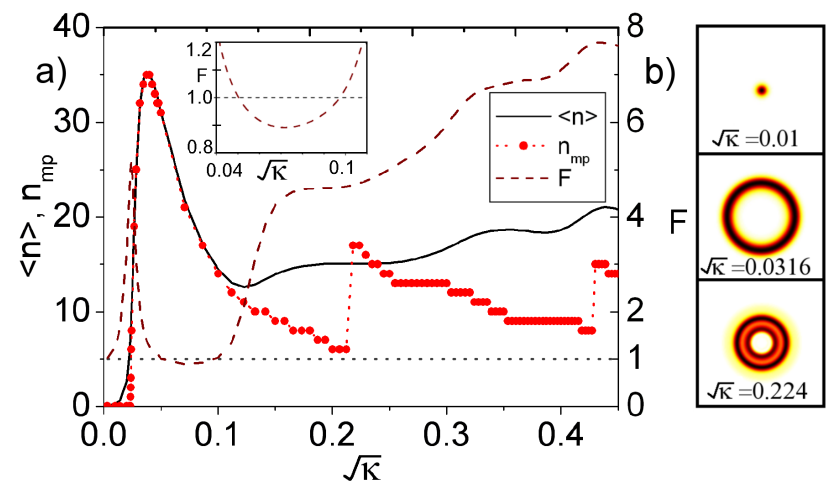

FIG. 4: (Color online) Changes in the resonator state (a) and corresponding Wigner functions (b) as the coupling $\kappa$ is increased for $\Delta E / e V_{d s}=-0.1, \omega / \Gamma=1, \gamma_{e x t} / \Gamma=0.001$ and $\bar{n}=0$. After an initial continuous transition $\left(\kappa \simeq 0.025^{2}\right)$, the system undergoes discontinuous transitions at $\kappa \simeq 0.22^{2}$ and $\kappa \simeq 0.44^{2}$. The inset in (a) shows the region where the Fano factor drops below unity. From top to bottom, the Wigner functions show: a fixed point state, a single limit cycle and two metastable limit cycles.

the SSET-resonator coupling is made stronger. A hallmark of the micromaser is the sequence of transitions which occur as the atom-cavity coupling is increased [10]. We find that the resonator undergoes a very similar sequence of transitions as the coupling is increased at fixed $\Delta E(<0)$, the first of which is continuous (for $|\Delta E|$ sufficiently small) and the rest discontinuous. An example of this behavior is shown in Fig. 4. As the coupling is increased from zero the resonator first undergoes a continuous transition between fixed point and limit cycle states. Within the limit cycle state $F$ drops below unity, but as $\kappa$ increases above $0.01, F$ grows sharply as a second metastable limit-cycle state begins to appear. For $\kappa \gtrsim 0.04$, the Wigner functions become more complicated, showing further metastable limit-cycles together with regions where parts of the Wigner function have negative values (indicating non-classicality of the resonator state). The relative weights of the different limit cycle states change with $\kappa$, leading to discontinuous transitions signalled by jumps in $n_{m p}$ as the most probable state of the resonator changes. In Fig. 4 only the first (continuous) transition shows sharp features in $\langle n\rangle$ and $F$ as we are far from the 'thermodynamic limit' [10] (we have chosen a relatively large $\gamma_{\text {ext }}$ to ensure that our truncation of the oscillator state space remains valid).

In conclusion, we have analyzed the dynamics of a resonator coupled to a SSET near the JQP resonance. The SSET-resonator system has a rich dynamics with many features in common with the micromaser including continuous and discontinuous transitions between resonator states and intrinsically quantum features such as non- classical steady-states. In practice, the resonator dynamics could be inferred from signatures in the current and current noise 19] of the SSET or, for a superconducting resonator, by coupling it to a transmission line 12]. The effects described are strongest when $\omega / \Gamma \sim 1$ and will require minimal thermal excitation $(\bar{n} \lesssim 1)$ for experimental observation of the non-classical features, implying high resonator frequencies. Similarities with the micromaser suggest the possibility of observing critical slowing down, hysteresis and quantum jumps in the resonator dynamics [6].

After submission of this paper, 20] was published which explores analogies between nano-electromechanical systems and laser physics in the slow-resonator limit. We thank T. Brandes, M. Blencowe and T. Harvey for useful discussions and acknowledge funding from the EPSRC.

[1] M.P. Blencowe, Phys. Rep. 395, 159 (2004).

[2] R.S. Knobel and A.N. Cleland, Nature 424, 291 (2003); M.D. LaHaye et al., Science 304, 74 (2004).

[3] A. Naik et al., Nature 443, 193 (2006).

[4] A.A. Clerk and S. Bennett, New J. Phys. 7, 238 (2005).

[5] T. Novotnỳ, A. Donarini and A.-P. Jauho, Phys. Rev. Lett. 90, 256801 (2003); D. Fedorets et al., Phys. Rev. Lett. 92166801 (2004); D.W. Utami et al., Phys. Rev. B 74014303 (2006); O. Usmani, Ya.M. Blanter and Y.V. Nazarov, /cond-mat/0603017 (unpublished).

[6] H. Walther et al., Rep. Prog. Phys. 69, 1325 (2006).

[7] I. Bargatin and M. Roukes, Phys. Rev. Lett. 91, 138302 (2003).

[8] M.S. Choi et al., Europhys. Lett. 53, 251 (2001).

[9] M.P. Blencowe, J. Imbers and A.D. Armour, New J. Phys. 7, 236 (2005).

[10] P. Filipowicz, J. Javanainen and P. Meystre, Phys. Rev. A 34, 3077 (1986).

[11] X.M.H. Huang et al., Nature 421, 496 (2003).

[12] A. Blais et al., Phys. Rev. A 69062320 (2004); A. Wallraff et al., Nature 431, 162 (2004).

[13] C. Flindt, T. Novotnỳ and A.-P. Jauho, Phys. Rev. B 70, 205334 (2004).

[14] The numerical method can also be used to explore the behavior of a more accurate (though less general) model of the SSET resonator system. Indeed, we have checked that including the position dependence of the quasiparticle rates does not qualitatively alter the results we present.

[15] T. Brandes and N. Lambert, Phys. Rev. B 67, 125323 (2003).

[16] S. M. Tan, J. Opt. B: Quantum Semiclassical Opt. 1, 424 (1999)

[17] D.A. Rodrigues and A.D. Armour, New J. Phys. 7, 251 (2005).

[18] H. Haken, Rev. Mod. Phys. 47, 67 (1975).

[19] C. Flindt, T. Novotnỳ, and A.-P. Jauho, Europhys. Lett. 69, 475 (2005).

[20] S. Bennett and A.A. Clerk, Phys. Rev. B 74, 201301 (2006). 